\title{
Segmentation of Lung Nodule in CT Images Based on Mask R-CNN
}

\author{
Menglu Liu \\ Department of Computer Science \\ and Technology \\ Ocean University of China \\ Qingdao, China \\ liumenglu@stu.ouc.edu.cn
}

\author{
Junyu Dong \\ Department of Computer Science \\ and Technology \\ Ocean University of China \\ Qingdao, China \\ dongjunyu@ouc.edu.cn
}

\author{
Xinghui Dong \\ Centre for Imaging Sciences \\ The University of Manchester \\ Manchester, M13 9PT, UK \\ xinghui.dong@manchester.ac.uk
}

\author{
Hui $\mathrm{Yu}$ \\ School of Creative Technologies \\ University of Portsmouth \\ Portsmouth, UK \\ hui.yu@port.ac.uk
}

\author{
Lin Qi* \\ Department of Computer Science and Technology \\ Ocean University of China \\ Qingdao, China \\ qilin@ouc.edu.cn
}

\begin{abstract}
Due to the low-quality of CT images, the lack of annotated data, and the complex shapes of lung nodules, existing methods for lung nodules detection only predict the center of the nodule, whereas the nodule size is a very important diagnostic criteria but is neglected. In this paper, we employed the powerful object detection neural network "Mask R-CNN" for lung nodule segmentation, which provides contour information. Because of the imbalance between positive and negative samples, we trained classification networks based on block. We selected the classification network with the hightest accuracy. The selected classification network was used as the backbone of the image segmentation network-Mask R-CNN, which performs excellently on natural images. Lastly, Mask R-CNN model trained on the COCO data set was fine-tuned to segment pulmonary nodules. The model was tested on the LIDC-IDRI dataset.
\end{abstract}

Keywords-deep learning, lung nodule segmentation, Mask $R$ CNN, LIDC-IDRI

\section{INTRODUCTION}

The incidence of lung cancer is rising because of the prolongation of life expectancy, acceleration of urbanization, ineffectiveness of anti-smoking campaigns, occupational exposure, environmental pollution, and genetic susceptibility. Lung cancer is one of the malignant tumors with the fastest increase in morbidity and mortality, and is one of the greatest threats to human's health and life. Lung cancer is the leading cause of cancer-related death worldwide. One out of every four deaths today is lung cancer. Therefore, how to effectively diagnose lung cancer has become a concern for all people. Early detection and diagnosis are the key to improving the survival rate of lung cancer patients.

Screening high risk individuals for lung cancer with lowdose computed tomography (CT) scans is now being studied in academic and industrial fields. The first step is the detection of pulmonary nodules in thoracic CT scans, based on which early

National Natural Science Foundation of China

*corresponding author treatment could significantly increase patient's chance of survival. In CT lung cancer screening, many millions of CT scans will have to be analyzed, which is an enormous burden for radiologists. Therefore there is a lot of interest to develop computer algorithms to optimize screening.

The pulmonary nodules are small, spheroidal, localized abnormal tissues that appear round or irregularly shadowed on CT. Pulmonary nodules are roughly divided into three categories: inflammatory nodules, benign nodules, and malignant nodules. Inflammatory nodules are caused by inflammation, anti-inflammatory treatment can be adopted; benign nodules can be followed up according to the doctor's guidance; malignant nodules require early surgical treatment. They have been regarded as crucial indicators of primary lung cancer, which has been the leading cause of cancer death in recent years.

Automated identification of the pulmonary nodules from thoracic CT scans is, however, among the most challenging tasks in computer-aided chest radiograph analysis for at least the following two reasons. First, the pulmonary nodules have large variations in sizes, shapes, and locations. Moreover, the contextual environments around them are often diversified for different categories of lung nodules. The boundary of lung nodules is rather vague, so the detection of nodules currently stays on locating the center of nodules. However, accurate prediction of nodule contour information is very important for cancer treatment.

Mask R-CNN [25] is one of the best instance segmentation model. It not only detects targets in the image, but also gives the predicted mask for each detected target. The model can be well extended to other tasks. So we proposed to transfer Mask R-CNN, which performs excellently on natural images, to segment lung nodules in CT images. In this paper, we approximate the pulmonary nodule on the $2 \mathrm{D}$ slice as a circle and train Mask R-CNN to predict both the nodule position and 

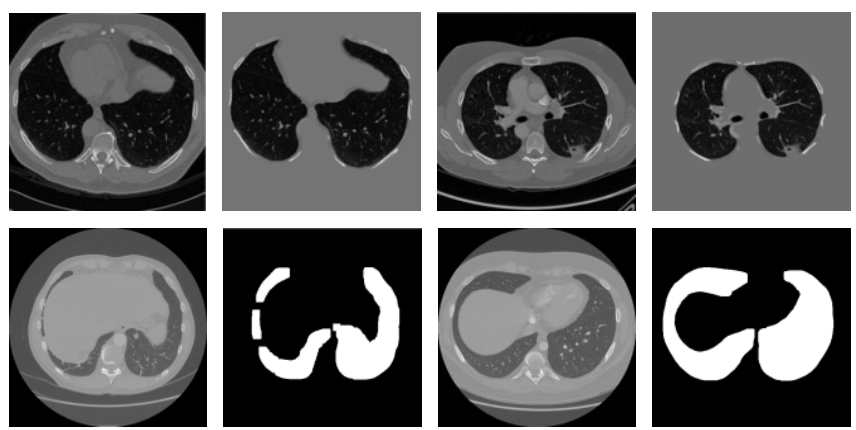

Fig. 1. LIDC-IDRI image examples. The first and third columns are $512 * 512$ CT images, and the second and fourth columns are corresponding lung region images.

the nodule contour (size).

To reduce interference, we segmented the lung area in advance and performed a series of experiments. There is a serious positive and negative sample imbalance in the pulmonary nodule image, and the proportion of nodule pixels in the lung area is less than $1 \%(0.00848)$. In order to solve this problem, we augmented the data by taking blocks around the nodules, and the blocks whose center pixel was nodule were marked as positive samples. This effectively increased the number of positive samples. We used these blocks to train the classification network which composed the backbone of the image segmentation network-Mask R-CNN. The Mask R$\mathrm{CNN}$ model trained on the $\mathrm{COCO}$ data set was fine-tuned to achieve segmentation of pulmonary nodules on CT images. We validated our method on the LUNA16 challenge.

\section{RELATED WORK}

\section{A. Computer-Aided Detection (CAD) Systems}

In order to perform lung cancer screening more effectively, Computer-Aided Detection (CAD) systems have been developed to assist radiologists in reading thousands of lung CT images. CAD is a software system, doctors use it to perform image processing and image analysis on the patient's medical image information, and detect the lesion. The purpose is to make the process of identifying lesions, especially small nodules, more objective and easy, and to improve the accuracy and efficiency of lesion detection. A CAD system for pulmonary nodule detection is typically divided into two phases: nodule candidate detection and false positive reduction. Many CAD systems have been proposed for nodule detection $[1,2]$. Traditional CAD systems can assist doctors to perform nodule detection to a certain extent, but the nodule has high variability in size, shape, and texture. Therefore traditional CAD systems cannot well capture image features of nodules, resulting in low accuracy of nodule detection.

In order to solve the problem of lung nodule detection, Armato et al. applied multiple gray-level thresholds on the volumetric lung regions to identify nodule candidates [9]. Choi and Choi proposed a novel three-dimensional shape-based feature descriptor to detect pulmonary nodules in CT scans. Nodule candidates were detected using multi-scale dot enhancement filtering in the segmented lung volume [10]. Li et al. proposed an improved detection method which combines a
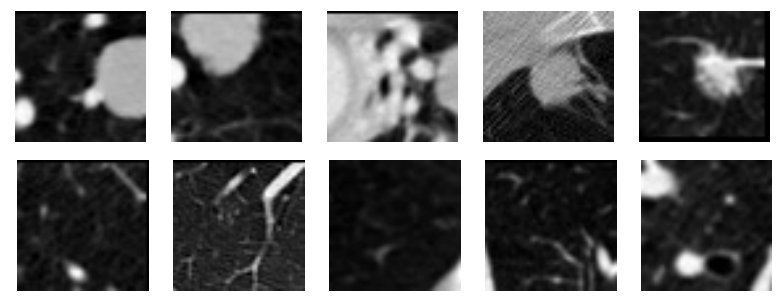

Fig. 2. 50*50 image blocks cut in the lung area. The first row is nodule blocks and the second row is normal lung blocks.

fuzzy integrated active contour model (FIACM), parametric mixture model (PMM), and a knowledge-based C-SVM (Costsensitive Support Vector Machines) classifier for the detection of nodules [11].

\section{B. Approaches using Deep Convolutional Neural Networks (CNNs)}

As convolutional neural networks $(\mathrm{CNN})$ achieved better results in natural image classification and detection, some studies have also attempted to apply convolutional neural networks to the detection of pulmonary nodules. SB et al. developed a double-matching method and an artificial visual neural network technique for lung nodule detection [12]. Lin et al. developed a neural-digital computer-aided diagnosis system, based on a parameterized two-level convolution neural network $(\mathrm{CNN})$ architecture and on a special multilabel output encoding procedure for lung nodule detection [13]. Both studies used pre-scan CAD systems to initially crop out nodule candidate regions from the radiographs. In the field of medical image, not only the detection task of lung nodules, but other similar tasks also tried to use deeper convolutional neural networks and transfer learning. Bar et al. explored using a AlexNet trained on the large image dataset ImageNet to detect Right Pleural Effusion and Enlarged heart conditions in radiographs [14]. Transfer learning played an important role when a task had very little training data. It can transfer the model parameters that have been trained on the big data set to own model.

Since AlexNet won the ImageNet Data Competition in 2012, convolutional neural networks have become popular, and CNN has achieved remarkable success in image and video processing [3]-[6]. A large number of researchers began to apply CNN to the field of medical image, and automatic lung nodule detection also began to use CNN. Setio et al. proposed a novel Computer-Aided Detection (CAD) system for pulmonary nodule detection using multi-view convolutional networks (ConvNets), for which representative features were automatically learnt from the training data [7]. Compared with the method using hand-crafted features, this method achieved higher accuracy. This method achieved a state of the art detection sensitivity of $85.4 \%$ at 1.0 false positive per subject on the benchmark of Lung Image Database Consortium-Image Database Resource Initiative (LIDC-IDRI) [8] dataset. This also demonstrated the effectiveness of convolutional neural networks in the detection of pulmonary nodules.

\section{The Region-Based CNN (R-CNN)}

The Region-based CNN (R-CNN) approach [15] can be said to be the pioneering work of using deep learning for object 
TABLE I. ClASSIFICATION ACCURACY OF DIFFERENT NETWORKS ON LIDC-IDRI DATASET

\begin{tabular}{|l|l|}
\hline \multicolumn{1}{|c|}{ Classification Network } & \multicolumn{1}{c|}{ Accuracy } \\
\hline LeNet & 0.950875 \\
\hline AlexNet & 0.958913 \\
\hline VGG16 & 0.959116 \\
\hline ResNet18 & 0.941875 \\
\hline ResNet20 & 0.94275 \\
\hline ResNet50 & 0.935875 \\
\hline ResNet101 & 0.965945 \\
\hline
\end{tabular}

detection. The first step in the R-CNN approach was to generate a manageable number of candidate object regions $[16,17]$. R-CNN extracted features using deep convolutional neural networks $[18,3]$ for each candidate region. R-CNN was extended $[20,21]$ to allow attending to RoIs on feature maps using RoIPool, leading to fast speed and better accuracy. Faster $\mathrm{R}-\mathrm{CNN}$ [22] has been around for nearly three years, but it is still one of the mainstream frameworks in the object detection field. The main innovation of Faster R-CNN is that it replaces the previous slow selective search algorithm with a fast neural network. Specifically, it introduces a Region Proposal Network (RPN). Faster R-CNN may not be the easiest and fastest way to object detection, but its performance is still the best at the moment.

Ding et al. [23] introduced a deconvolutional structure to Faster Region-based Convolutional Neural Network (Faster R$\mathrm{CNN}$ ) that incorporated an ImageNet pre-trained VGG-16 model [24] to perform candidate detection on axial slices. Then, a three-dimensional DCNN was used for the subsequent false positive reduction. Their methodology yielded a FROC-score of 0.893 on the LUNA dataset (topping the LUNA16 leaderboard in May 2017). However, these methods based on R-CNN merely locate nodules and do not give the nodule contour information.

\section{DATA PREPROCESSING}

\section{A. Database}

The Lung Image Database Consortium image collection (LIDC-IDRI) consists of diagnostic and lung cancer screening thoracic computed tomography (CT) scans with marked-up annotated lesions.

Seven academic centers and eight medical imaging companies collaborated to create this data set which contains 1018 cases. Each subject includes images from a clinical thoracic CT scan and an associated XML file that records the results of a two-phase image annotation process performed by four experienced thoracic radiologists. In the initial blindedread phase, each radiologist independently reviewed each CT scan and marked lesions belonging to one of three categories (nodule $>=3 \mathrm{~mm}$, nodule $<3 \mathrm{~mm}$ and non-nodule $>=3 \mathrm{~mm}$ ). In the subsequent unblinded-read phase, each radiologist independently reviewed their own marks along with the anonymous marks of the three other radiologists to render a final opinion. The goal of this process was to identify as completely as possible all lung nodules in each CT scan
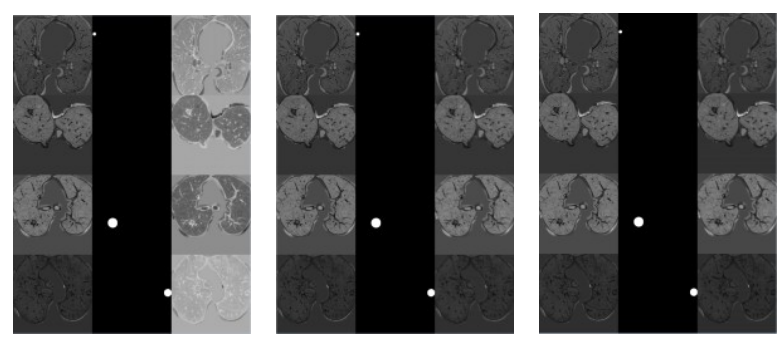

Fig. 3. U-Net segmentation result on lung image. The first column in each image is the input CT image, the second column is the corresponding mask, and the third column is the prediction result.

without requiring forced consensus.

The annotations were provided by the Lung Nodule Analysis (LUNA) challenge as part of the 2016 IEEE International Symposium on Biomedical Imaging. The LUNA challenge is therefore a completely open challenge. LUNA2016 excluded scans with a slice thickness greater than $2.5 \mathrm{~mm}$. In total, $888 \mathrm{CT}$ scans are included. LUNA2016 challenge consists of all nodules $>=3 \mathrm{~mm}$ accepted by at least 3 out of 4 radiologists. This paper ends up using data set provided by LUNA2016. The images in the data set were shown in Fig. 1. Dataset contains 512*512 CT images.

\section{B. Data Preprocessing}

The image provided by LUNA2016 is a series of CT slices. For the segmentation of pulmonary nodules, many slices are not required. CT image is different from the normal image. Therefore, a series of processing must be done before use.

- Slice Selection: CT images consist of a series of 2D slices. There are several hundred slices per CT scan. Not all slices have nodules. For nodule segmentation task, we only need slices with nodules. Since the nodule position is defined by the sphere and the nodule is irregularly shaped, the section near the edge of the sphere may not contain nodular tissue. Using such slices may produce false positives that lead to contaminate training sets. For this segmentation task, there may be an optimal number of contained slices. But for the sake of simplicity, we only took 3 slices and chose only the largest slice near the center of the nodule. Masks will be created for these slices based on the nodule size given in annotations.csv. Two files were acquired for each patient: a set of images and a set of corresponding nodule masks.

- Lung Segmentation: To narrow the scope of detection, lung segmentation were performed as a preliminary step. All experiments were then performed in the lung area. The lungs are the primary organs of the respiratory system in the human body, and consist of a left and a right lung, situated within the thoracic cavity of the chest. Since lungs also have relatively bright components such as blood vessels and nodules, the entire surface will become smooth by performing operations such as pore filling and morphological corrosion expansion. Segmented lung area was shown in Fig. 1. 

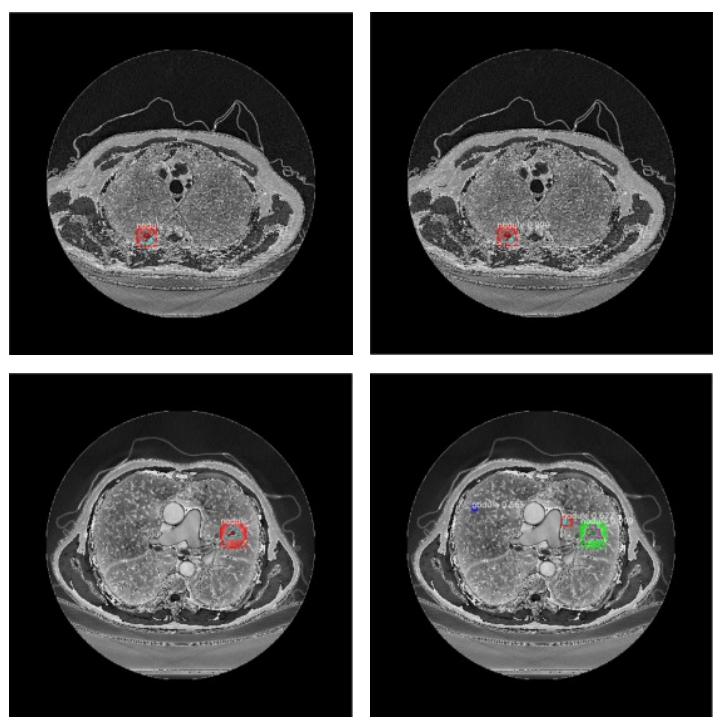

Fig. 4. Mask R-CNN's segmentation result graph, the first column is the input image, and the second column is the segmentation image. For the convenience of viewing, we marked the label in the input image. The different nodules segmented by Mask R-CNN were represented by different colors in the segmentation image.

- Normalization: Hounsfield value is the unit to represent the relative density of tissues and organs on CT images. In most of the lung CT images, the Hounsfield value varies from -1000 to 1000 . After analysis, the meaningful pixel values for nodule judgment are $[-1024,800]$. The final image pixel values were clipped to $[-1024,800]$, then zoomed to $[0,1]$.

- More importantly, the training dataset has extremely high false positive to true positive ratio. In order to solve this problem, we cut the lung region of the original image into $50 * 50$ small blocks. Nonoverlapped normal patches in the lungs were used as negative samples. Near nodule, we took a $50 * 50$ block every ten pixels. We identified the block whose center pixel is a nodule pixel as a positive sample. Finally, we expanded the positive sample more than 20 times. The block images were shown in Fig. 2.

\section{METHODS AND EXPERIMENTS}

\section{A. Mask R-CNN backbone selection}

Mask R-CNN backbone is classification and detection network. Before using Mask R-CNN for pulmonary nodule segmentation, we first discuss what kind of network should be selected as the backbone of Mask R-CNN.

1) Classification Network: A convolutional neural network is a multilayer neural network that excels at dealing with the related machine learning problems of images, especially large images. Through a series of methods, the convolutional network successfully reduces the dimension of image with large amounts of data, and finally enables it to be trained. The classic LeNet was born in 1998. However, the sharpness of CNN began to be overshadowed by manually

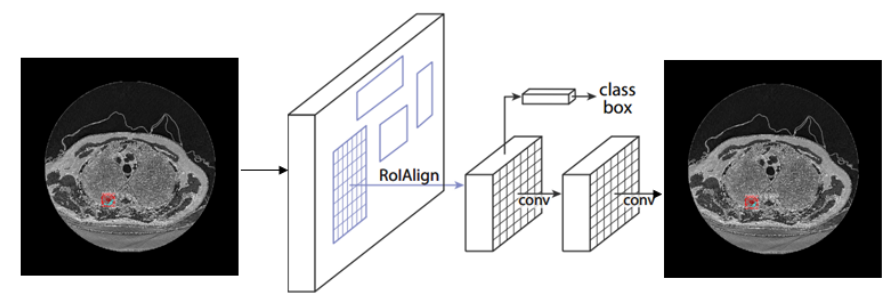

Fig. 5. Mask R-CNN overall framework. For ease of observation, we marked ground truth in the input image. The output image demonstrates the segmentation result. In this figure, Mask R-CNN perfectly segmented the nodule.

designed features such as SVM. With the introduction of ReLU and dropout, and the historical opportunities brought by GPUs and big data, $\mathrm{CNN}$ ushered in historical breakthroughs in 2012 - AlexNet, followed by VGG, GoogLeNet, and ResNet. They are all outstanding players in the ImageNet Large Scale Visual Recognition Challenge (ILSVRC) competition over the years. CNN has become the only choice for computer vision applications. It not only plays an important role in the field of natural images, but also is crucial in the field of medical image. Classification networks that perform well in natural images are equally effective in classifying lung nodules. This is a binary problem. In order to reduce the interference, we extracted the lung area and removed the part outside the lung. In the data set used, the proportion of nodule pixels to the number of pixels in the lungs is less than $1 \%$. There is a serious imbalance between positive and negative sample proportions. For this reason, we intercepted $50 * 50$ image blocks and finally made the ratio of positive and negative samples $1: 1$. We has 24,000 images in the training set, and 8000 images in the verification set and the test set respectively. We used image blocks to train different classification networks. The results were shown in TABLE I. As can be seen from the table, for our data, ResnetNet 101 has the highest accuracy relative to other classification networks. Therefore, we eventually selected ResNet101 as Mask R-CNN classification network.

2) Detection Network: U-Net [19] plays an important role in cell segmentation. U-Net outperforms the prior best method (a sliding window convolutional network) on the ISBI challenge for segmentation of neuronal structures in electron microscopic stacks. U-Net consists of a contracting path to capture context and a symmetric expanding path that enables precise localization. We used our lung CT images to train UNet. The results were shown in Fig. 3. The first column of each image in the figure is the input image, the second column is the nodule mask, and the third column is the segmentation result. As shown in the figure, U-Net does not perform well on our dataset. It did not properly segment the nodules but instead segmented the lungs. We think it may be because the nodules are too small compared to the lung. Compared to U-Net, a top-down architecture with skip connections, where predictions are made on the finest level, 


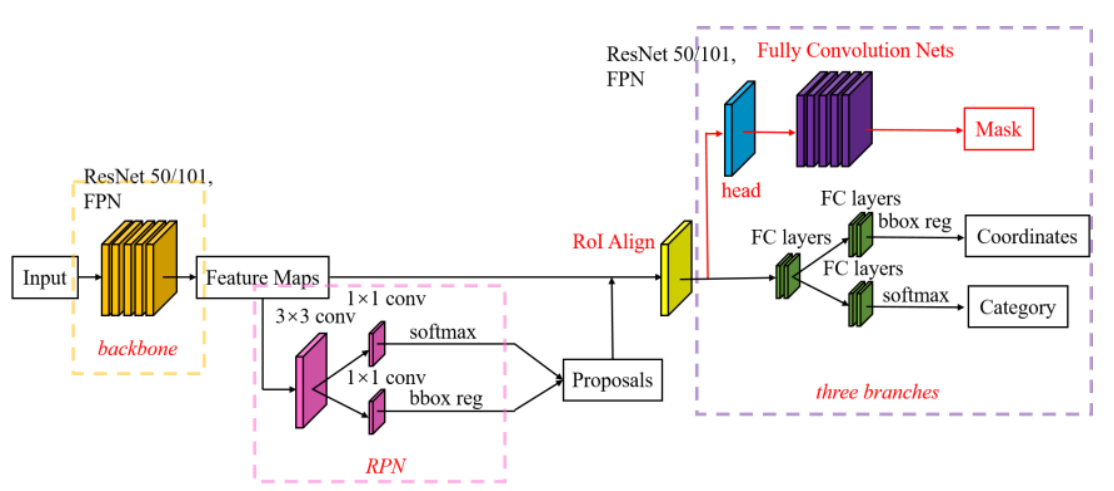

Fig. 6. Mask R-CNN detailed framework

FPN [26] has a similar structure but leverages it as a feature pyramid, with predictions made independently at all levels. FPN shows significant improvements over all exisiting stateof-the-art methods and can achieve higher accuracy. We eventually selected FPN as Mask R-CNN detection network.

\section{B. Mask R-CNN}

Mask R-CNN is a small, flexible generic object instance segmentation framework. It not only detects targets in the image, but also gives a high-quality segmentation result for each target. It is extended on the basis of Faster R-CNN, and adds a new branch for predicting an object mask which is parallel with bounding box recognition branch. The network is also easily extended to other tasks, such as estimating a person's posture, that is, person keypoint recognition. The framework has achieved the best results in some of the COCO's challenging tasks, including instance segmentation, bounding-box object detection, and person keypoint detection. The overall framework of Mask R-CNN was shown in Fig.5.

This paper used FPN and ResNet101 as the backbone of Mask R-CNN. The detailed framework of Mask R-CNN was shown in Fig.6 [27]. Mask R-CNN is divided into two stages, the first stage is RPN (Region Proposal Networks). RPN is a new proposal generation network from Faster RCNN, it replaces the selective search method in the previous R-CNN and Fast R-CNN, and integrates all the content in one network, which greatly improves the detection speed. The second stage has two parallel branches, one is the bounding box branch for detection, the bounding box branch contains classification and bounding box regression. The other is the mask branch for segmentation. Mask R-CNN defines the loss function as follows:

$$
L=L_{c l s}+L_{b o x}+L_{m a s k}
$$

Annotating biomedical images is not only tedious, costly and time consuming, but also need specialty-oriented knowledge and skills. Therefore, a large number of annotated biomedical images are not easily accessible. This paper transferred the model trained on the COCO data set. We used $512 * 512$ lung CT images to fine tune existing models. The training set used 2134 CT images, the verification set used 711 images, and the test set used 711 images. In order to increase the amount of images, translation and rotation augmentation were conducted. The learning rate was initialized as 0.0001 . The momentum was set to 0.9 .

We used two methods to train Mask R-CNN: (1)use our own data to fine-tune all layers directly on the pretrained model of COCO data set. The accuracy obtained is $0.7334 \mathrm{mAP}$ (Mean Average Precision). (2)train network heads first and then fine tune ResNet stage 3 and up; lastly, fine tune all layers. The accuracy obtained is $0.7965 \mathrm{mAP}$. Mask R-CNN segmentation result was shown in Fig. 4. Because only professional doctors can identify nodules from CT images, in order to enable people without professional knowledge to know the location of the nodules at a glance, we specifically marked the nodules in the input image so that people can easily compare input image and segmentation result. From the results, Mask R-CNN accurately found the position of the nodule and gave corresponding contour information. Although some of the non-nodule were treated as nodule, the segmentation task is quite difficult for the object such as the nodule which is very small, so such errors are acceptable. This paper improved the existing instance segmentation network and applied it to the segmentation of lung nodules in CT images. The contour information of the nodules was given for the first time. This work is very valuable for the early diagnosis and treatment of lung cancer.

\section{CONCLUSION AND FUTURE WORK}

Lung cancer is the leading cause of cancer death. Early detection and treatment are essential to improve 5-year survival. The main method for the early diagnosis of lung cancer is to detect pulmonary nodules. Due to the low-quality of CT images, the scarcity of annotated data, the complex shapes and unclear contours of pulmonary nodules, the current detection task for lung nodules only remains at locating the center of the nodule. This paper used the latest Mask R-CNN to segment lung nodules for the first time. After a series of comparative experiments, ResNet101 and FPN were selected as the backbone of Mask R-CNN. Experimental results not only locate the location of nodules, but also provide nodule contour information. It provided more detailed information for cancer treatment. The experimental results have been validated on the LIDC-IDRI data set and achieved desired accuracy.

The CT image is a 3D image. 3D contexts play an import role in recognizing nodules. A 3D network performs better than a $2 \mathrm{D}$ network as it also captures the vertical information 
of nodules. We now realized the segmentation of nodules on $2 \mathrm{D}$ images, using the $2 \mathrm{D}$ network structure. Next we will extend the $2 \mathrm{D}$ network structure to $3 \mathrm{D}$ to achieve more accurate segmentation of nodules on 3D CT images.

\section{ACKNOWLEDGMENT}

This work was supported by the National Natural Science Foundation of China (NSFC) (No.61501417) and the Ph. D. Program Foundation of Ministry of Education of China (No.20 120132110018).

\section{REFERENCES}

[1] E. L. Torres, E. Fiorina, F. Pennazio, and et al. Large scale validation of the m51 lung cad on heterogeneous ct datasets. Medical Physics,42(4):1477-1489, 2015.

[2] A. A. A. Setio, A. Traverso, T. Bel, and et al. Validation, comparison, and combination of algorithms for automatic detection of pulmonary nodules in computed tomography images: the luna16 challenge. In arXiv:1612.08012, 2016.

[3] A. Krizhevsky et al., "Imagenet classification with deep convolutional neural networks," in Proc. Adv. Neural Inf. Process. Syst., 2012,pp. 1097-1105.

[4] K. He et al., "Deep residual learning for image recognition," 2015.[Online]. Available: arXiv:1512.03385.

[5] X. Qi et al., "Semantic segmentation with object clique potential," in Proc.IEEE Int. Conf. Comput. Vis., 2015, pp. 2587-2595.

[6] S. Ji et al., "3d convolutional neural networks for human action recognition," IEEE Trans. Pattern Anal. Mach. Intell., vol. 35, no. 1, pp. 221-231,Jan. 2013

[7] A. A. A. Setio et al., "Pulmonary nodule detection in ct images: False positive reduction using multi-view convolutional networks," IEEE Trans.Med. Imag., vol. 35, no. 5, pp. 1160-1169, Mar. 2016

[8] S. G. Armato III et al., "The lung image database consortium (lidc) and image database resource initiative (idri): A completed reference database of lung nodules on ct scans," Med. Phys., vol. 38, no. 2, pp. 915931,2011.

[9] Rd A S, Giger M L, Moran C J, et al. Computerized detection of pulmonary nodules on CT scans.[J]. Investigative Radiology, 1999, 19(4):1303-1311.

[10] Choi W J, Choi T S. Automated pulmonary nodule detection based on three-dimensional shape-based feature descriptor[J]. Computer Methods \& Programs in Biomedicine, 2014, 113(1):37.

[11] Li B, Chen K, Tian L, et al. Detection of pulmonary nodules in CT images based on fuzzy integrated active contour model and hybrid parametric mixture model[J]. Computational and mathematical methods in medicine, 2013, 2013(1):515386.

[12] S.-C. Lo, S.-L. Lou, J.-S. Lin, M. Freedman, M. Chien, and S. Mun. Artificial convolution neural network techniques and applications for lung nodule detection. IEEE Transactions on Medical Imaging, 14(4), 1995.

[13] J.-S. Lin, S.-C. Lo, A. Hasegawa, M. Freedman, and S. Mun. Reduction of false positives in lung nodule detection using a two-level neural classification. IEEE Transactions on Medical Imaging, 15(2):206-217, 1996.

[14] Y. Bar, I. Diamant, L. Wolf, and H. Greenspan. Deep learning with nonmedical training used for chest pathology identification. SPIE, 9414, 2015.

[15] R. Girshick, J. Donahue, T. Darrell, and J. Malik. Rich feature hierarchies for accurate object detection and semantic segmentation. In CVPR, 2014.

[16] J. R. Uijlings, K. E. van de Sande, T. Gevers, and A. W. Smeulders. Selective search for object recognition. IJCV, 2013.
[17] J. Hosang, R. Benenson, P. Dollar, and B. Schiele. What ' makes for effective detection proposals? PAMI, 2015.

[18] Y. LeCun, B. Boser, J. S. Denker, D. Henderson, R. E. Howard, W. Hubbard, and L. D. Jackel. Backpropagation applied to handwritten zip code recognition. Neural computation, 1989.

[19] Ronneberger, Olaf, P. Fischer, and T. Brox. "U-Net: Convolutional Networks for Biomedical Image Segmentation." International Conference on Medical Image Computing and Computer-Assisted InterventionSpringer, Cham, 2015:234-241.

[20] K. He, X. Zhang, S. Ren, and J. Sun. Spatial pyramid pooling in deep convolutional networks for visual recognition. In ECCV. 2014.

[21] R. Girshick. Fast R-CNN. In ICCV, 2015.

[22] S. Ren, K. He, R. Girshick, and J. Sun. Faster R-CNN: Towards realtime object detection with region proposal networks. In NIPS, 2015.

[23] J. Ding, A. Li, Z. Hu, and L. Wang (2017): "Accurate Pulmonary Nodule Detection in Computed Tomography Images Using Deep Convolutional Neural Networks," MICCAI 2017.

[24] K. Simonyan and A. Zisserman (2015): "Very Deep Convolutional Networks for Large-Scale Image Recognition," ICLR 2015.

[25] He K, Gkioxari G, Dollár P, et al. Mask R-CNN[C]// IEEE International Conference on Computer Vision. IEEE, 2017:2980-2988.

[26] Lin T Y, Dollar P, Girshick R, et al. Feature Pyramid Networks for Object Detection[J]. 2016:936-944.

[27] Jiong. https://blog.csdn.net/jiongnima/article/details/79094159, 2018$01-21$. 